Trang

SECCIÓN: Problemas espaciales contemporáneos
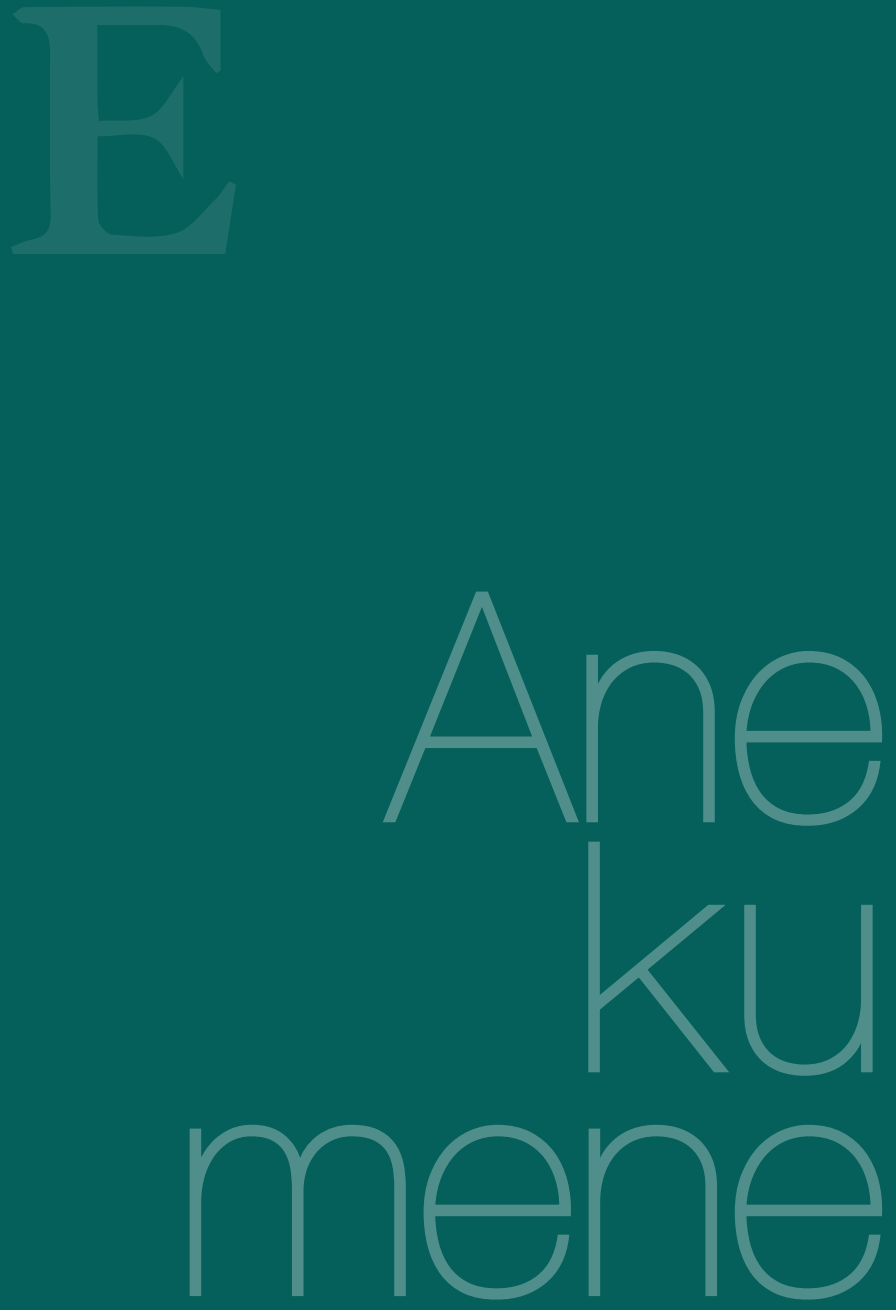


\section{Prácticas sociales y ciudadanía en espacios públicos de Bogotá: caso zona El Tintal}

Social Practices and Citizenship in Public Spaces in Bogota: El Tintal Case

\section{Práticas sociais e cidadania em espaços públicos de Bogotá: Caso zona El Tintal}

Diego Ariza Coy*

\begin{abstract}
Resumen
El presente artículo expone un análisis socioespacial sobre el sector de El Tintal, en el occidente de Bogotá. Allí se interpretan prácticas sociales asociadas con el ejercicio de la ciudadanía por parte de sujetos que habitan el espacio público. A través de las prácticas sociales, el espacio habitado se reproduce por un proceso dialéctico continuo que obedece a las mismas dinámicas en las que se desarrollan las sociedades; por tal motivo, la producción del espacio en las ciudades adquiere una connotación social si se entiende como acto del habitar. Esta concepción, vinculada con la práctica social sobre el espacio público, es la fuente de indagación ideal para un trabajo investigativo en torno a la ciudad, ya que se evidencian valores, actitudes y estructuras cognitivas de sus habitantes como expresión de sus procesos de socialización.
\end{abstract}

\section{Palabras clave}

Prácticas sociales; habitar; espacio público; ciudadanía

* Docente de Ciencias Sociales en el Colegio Ciudadela Educativa de Bosa, IED. 


\section{Keywords Abstract}

Social Practices; Dwelling; Public Space; Citizenship
In this paper we present a socio-spatial analysis of the sector of El Tintal, in western Bogota, by interpreting social practices associated with the exercise of citizenship by individuals inhabiting public spaces. Through social practices, inhabited spaces are reproduced by a continuous dialectical process that follows the same dynamics of social development; therefore, the production of space in cities acquires a social connotation when it is understood as an act of inhabiting. This conception, which is linked to social practices over public space, is the ideal source of inquiry for an investigative work about the city, as it reveals the values, attitudes and cognitive structures of its inhabitants as an expression of their socialization processes.

\section{Palavras-chave}

práticas sociais; habitar; espaço público; cidadania

\section{Resumo}

O presente artigo expõe uma análise socioespacial sobre o setor de El Tintal, no ocidente de Bogotá. Nesta, abordam-se práticas sociais associadas com o exercício da cidadania por parte de sujeitos que habitam o espaço público. A traves das práticas sociais, o espaço habitado se reproduz por um processo dialético continuo que obedece às mesmas dinâmicas nas que se desenvolvem as sociedades. Por isso, a produção do espaço nas cidades adquire uma conotação social, se é entendida como um ato do habitar. Esta concepção, vinculada com a prática social sobre o espaço público, é a fonte de indagação ideal para um trabalho de pesquisa sobre a cidade, pois evidencia valores, atitudes e estruturas cognitivas dos seus habitantes como expressão dos seus processos de socialização. 


\section{Espacio público, prácticas sociales y ciudadanía}

La ciudad es un espacio de vida construido para la acción, donde los sujetos son emisores y receptores de prácticas ideológicas presentes en todo proceso de praxis social (Lefebvre, 1968, citado en Castells, 1976). Dentro de ella, el espacio público aparece como escenario urbano ${ }^{1}$ donde se producen nuevas prácticas sociales que denotan la interacción entre individuos o grupos con características heterogéneas a través del encuentro, el debate y el consenso frente a la tensión entre intereses generales y particulares de la población.

El espacio público contiene la expresión, la comunicación y el desarrollo de la vida social de los seres que habitan la ciudad. Su importancia investigativa emerge cuando se analizan las acciones del sujeto junto con los discursos por los cuales se generan significados socialmente producidos ${ }^{2}$. Se busca entonces identificar, desde las prácticas sociales que allí acontecen, fragmentos de la subjetividad que denotan una experiencia espacial y que constituyen un acto del habitar ${ }^{3}$. Lo anterior obedece a una doble connotación, primero, se hace referencia a la apropiación del espacio por parte del hombre que se afirma y se afianza con una manera particular de sentirse en el mundo; segundo, se alude al ejercicio de asentamiento sobre un espacio donde este puede desplegar todo su potencial creativo, para configurarlo con el fin de establecer sentidos de identificación y pertenencia. Desde esta apreciación se asevera que "no se habita en un lugar, sino que se hace un lugar donde se habita” (Yory, 2007, p. 295).

De esta forma, los espacios públicos se definen como espacios del habitar gracias a su condición de exterioridad ${ }^{4}$, aspecto clave para identificar formas de apropiación espacial vinculadas con la experiencia del sujeto que evidencian la construcción social del espacio. Dicha apropiación emerge desde prácticas sociales que son reconocidas como formas institucionalizadas de relación social, como procedimientos, métodos o técnicas cualificadas realizadas por agentes sociales, que permiten establecer campos para la acción y la realización de los seres humanos (Giddens, 1990).

Desde las condiciones del habitar la ciudad y de apropiar sus espacios públicos, las prácticas sociales son un conjunto de posibilidades y de prohibiciones para la acción del individuo que se producen en relaciones de

1 El término escenario urbano se retoma de Alicia Lindón (2010), quien lo define como fragmento de la ciudad que condensa los movimientos cotidianos de los sujetos en términos de espacio y de tiempo. Estos escenarios tienen un fuerte anclaje con el lugar en la medida que reflejan diferentes maneras de usar, conquistar, apropiar, defender y explorar la ciudad a través de la acción del sujeto.

2 La acción y el discurso son herramientas que usa el sujeto para mostrarse en público y expresar sus concepciones frente a su relación con el mundo y con los otros con quienes interactúa (Arendt, 1993). Para esta investigación, la acción se relaciona con las prácticas que pueden ser observadas por los otros y el discurso; por su parte, permite anunciar aquello que se hace o lo que se puede hacer sobre el espacio público,

3 Este proceso se puede definir en términos de lo que Carlos Yory (2007) denomina como espaciación; es decir, como el acto de apertura que el hombre ejerce sobre el espacio al momento de construir o fundar los lugares en función de sus necesidades particulares, que le garantizan la creación de escenarios para el habitar en pro de su propio beneficio.

4 La condición de exterioridad se atribuye a aquellos espacios abiertos donde el habitante de la ciudad tiene la posibilidad del encuentro con el otro o con lo desconocido. Son escenarios ideales para dar cuenta de lo que ocurre en la ciudad a partir de las acciones del sujeto y las tramas de significado generadas por la práctica social (Lindón, 2010). continuidad y cercanía para su constitución en lugares (De Certeau, 1996). Esta dinámica influye en la formalización de normas para la interacción social que regulan y organizan las relaciones entre los seres humanos. El conocimiento social así formulado permite a los individuos hacer uso de una serie de técnicas necesarias para manipular o influir de manera sustantiva en la realización de actividades sociales (oportunidades o situaciones espaciotemporales concretas que reglamentan las relaciones entre los seres humanos) y en los objetos materiales necesarios para producir la acción (los recursos presentes en un espacio determinado).

Las prácticas que despliega cada sujeto están encadenadas o entretejidas en secuencias de acciones, orientadas a alcanzar algo; al mismo tiempo, estas se relacionan con las de otro con el que se comparte determinado espacio. Precisamente, como los espacios públicos han sido desarrollados para facilitar el encuentro, la socialización y la interacción con un grupo de extraños a partir de reglas que moldean y regulan la acción humana (Páramo, 2007), su ocupación se encuentra condicionada por la interacción entre los sujetos y la capacidad que estos tienen de comunicarse entre sí. Estos procesos de interacción permiten establecer conexiones de cercanía hacia los otros a través de la coapropiación, acto mediante el cual el sujeto interactúa con el espacio, unido a la formación de significados particulares y asociado con el tipo de experiencia espacial que este desarrolle.

Desde aquí emana la condición ciudadana de habitar el espacio público a través del desarrollo de actos de presencia y visibilidad mutua, donde la pluralidad de perspectivas permite la posibilidad del encuentro de saberes y haceres relacionados con la vida en la ciudad. La condición del ser ciudadano (Sánchez, 2007) implica ante todo un proceso de reconocimiento por parte del individuo como sujeto social, que a su vez tiene en consideración al otro como sujeto en dinámicas de coexistencia.

En este sentido, la importancia del espacio público como escenario para el reconocimiento del sujeto y de su condición como ser social vincula a la ciudadanía como el catalizador que dinamiza formas de acción colectiva hacia la construcción de la vida pública en las ciudades, donde se producen expresiones cotidianas relacionadas con la vecindad, la comunicación, la diversidad y la convivencia, propias de determinada experiencia espacial. Los procesos de aprehensión hacia el espacio público implican, desde el ejercicio de la ciudadanía, una correspondencia y coapropiación que permitan no solo la interacción constante con otros agentes, sino también el ejercicio del sujeto en propiedad, convirtiendo al espacio en un habitáculo para su realización de ser y estar en el mundo.

Para que esta apropiación (entrar en propiedad) sea posible, se requiere que el sujeto a partir de su contacto con otros logre generar niveles de reflexividad, agenciamiento y sociabilidad ${ }^{5}$, que le permitan recrear prácticas sociales constituidas por la puesta en común de un acervo de conocimientos compartidos, que dan identidad tanto al grupo social

5 La reflexividad implica el autoreconocimiento del sujeto como productor y receptor de significados construidos socialmente. Por su parte, el agenciamiento y la sociabilidad son los elementos por los cuales el sujeto define la intencionalidad y la autodeterminación de sus acciones, unido con la capacidad de entablar interacciones sociales en contacto con otros. 
como al espacio en el que cohabita. De esta manera, un espacio público construido socialmente se constituye en un lugar que alberga y contiene la vida del hombre, desde sus diferentes elementos físicos hasta los componentes subjetivos que potencian el significado.

La producción de espacios públicos va ligada con lo que significa habitarlos y con el encontrar una forma de ser y estar en el mundo. Este proceso puede desprenderse de la realización de prácticas sociales que configuran el desarrollo de la vida social. Así, el espacio público se va configurando desde la articulación de reglas, normas, objetos, marcas, percepciones y subjetividades desarrolladas a través de las acciones en el sujeto y que son instituidas mediante una práctica social concreta. El habitar construye el espacio y, en el caso de los espacios públicos, esta condición se presenta de forma más espontánea a causa de la ocupación, apropiación y significación que sus habitantes hacen de él por medio de la interacción y práctica social.

\section{Observar, describir e interpretar el espacio público desde las prácticas sociales}

En los espacios públicos acontecen diversos tipos de prácticas sociales relacionadas con procesos de participación y expresión sociocultural, en las que se incluye el ejercicio de la ciudadanía como expresión de coexistencia y convivencia ante la diversidad y la multiplicidad social. En esta investigación se realizó un análisis sobre los espacios públicos de la Biblioteca Pública Mayor El Tintal y el Parque Metropolitano Urbano de El Tintal, emplazados entre la Avenida Ciudad de Cali y la Carrera 88 (de oriente a occidente) y entre la Calle 6 y la Calle $6 \mathrm{C}$ (de sur a norte), ubicados en la localidad de Kennedy al occidente de la ciudad de Bogotá.
En estos espacios públicos se analizaron formas de interacción humana a través de la constitución de lugares para el habitar. Se pretendió identificar, entre diversas prácticas sociales de los sujetos que habitan estos escenarios, formas de interpretar y concebir los espacios vividos desde la subjetividad a partir de un interrogante central: ¿cómo las prácticas sociales, desarrolladas por los sujetos que habitan la Biblioteca Pública y el Parque Metropolitano Urbano de El Tintal, contribuyen a la construcción de lugares para la socialización y el ejercicio de la ciudadanía desde la apropiación de sus espacios públicos? (ver mapa 1).

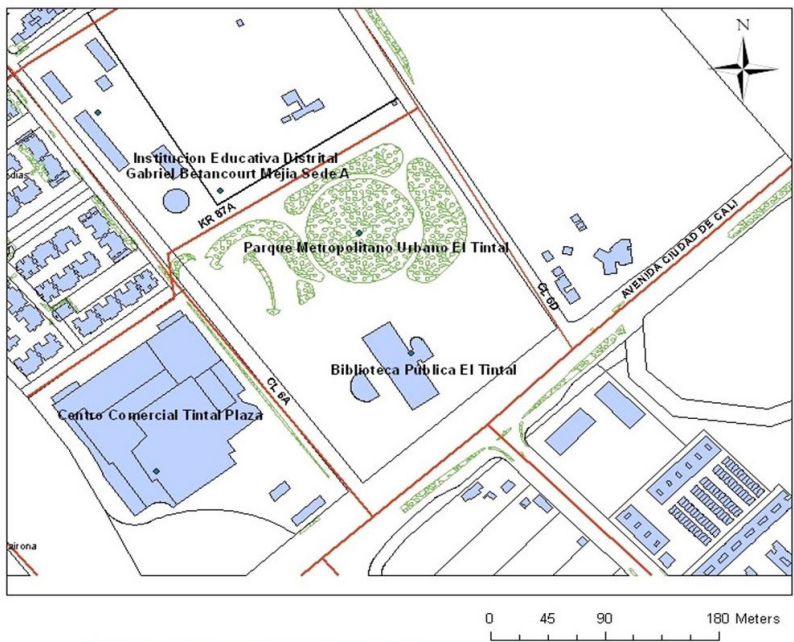

Mapa 1. Espacios públicos de la Biblioteca y el Parque Metropolitano Urbano de El Tintal.

Fuente: elaboración propia.

La investigación se enmarcó dentro de un horizonte epistemológico de corte cualitativo-interpretativo, asociado con la sociología urbana y enfocada desde la etnografía para comprender el uso y la apropiación de los espacios públicos en la zona de estudio. A partir de tales parámetros se abordaron diversos tipos de estrategias para abarcar de forma ampliada los objetivos del trabajo (ver tabla 1).

Tabla 1. Matriz de estrategia metodológica.

\begin{tabular}{|c|c|c|c|}
\hline Objetivo & $\begin{array}{l}\text { Técnica de } \\
\text { investigación }\end{array}$ & Instrumentos de investigación & Finalidad \\
\hline $\begin{array}{l}\text { Caracterizar prácticas sociales desarrolladas por } \\
\text { los sujetos en relación con las maneras como se } \\
\text { ocupan y usan los espacios públicos. }\end{array}$ & Observación flotante & $\begin{array}{l}\text { Diario de campo y registro } \\
\text { fotográfico. }\end{array}$ & $\begin{array}{l}\text { Establecer características espaciales y } \\
\text { poblacionales de la zona. }\end{array}$ \\
\hline $\begin{array}{l}\text { Interpretar formas de apropiación sobre el espacio } \\
\text { público a partir de la construcción de referentes } \\
\text { socioespaciales y su relación con la condición } \\
\text { ciudadana de los sujetos. }\end{array}$ & $\begin{array}{l}\text { Observación } \\
\text { participante }\end{array}$ & $\begin{array}{l}\text { Entrevista cualitativa enfocada } \\
\text { (Bonilla y Rodríguez, 1995). } \\
\text { Encuesta estructurada semiabierta } \\
\text { (López, 1998). }\end{array}$ & $\begin{array}{l}\text { Identificar relaciones entre práctica } \\
\text { social y cotidianidad en la experiencia } \\
\text { particular de los sujetos. }\end{array}$ \\
\hline $\begin{array}{l}\text { Representar tipificaciones que expresan formas de } \\
\text { habitar el espacio público en la ciudad. }\end{array}$ & $\begin{array}{l}\text { Codificación de } \\
\text { información }\end{array}$ & $\begin{array}{l}\text { Cartografía digitalizada (Valencia, } \\
\text { 2009). }\end{array}$ & $\begin{array}{l}\text { Analizar formas de apropiación sobre } \\
\text { los espacios públicos en relación con } \\
\text { el habitar la ciudad. }\end{array}$ \\
\hline
\end{tabular}

Fuente: elaboración propia. 
La implementación de la estrategia metodológica se orientó hacia la interpretación de prácticas sociales en los espacios públicos descritos a partir de dos referentes de análisis a considerar:

I. La contextualización y la caracterización de la zona en aspectos históricos, normativos y espaciales para establecer su conformación y evolución histórica hasta constituirse en espacio público, en el cual se generan prácticas sociales relacionadas con el habitar.
II. La caracterización de los sujetos desde el tipo de prácticas sociales que desarrollan y establecen las formas de concebir y apropiar el espacio público hasta su constitución en lugar para el habitar.

El resultado de este proceso llevó a que se determinara la transformación espacial del sector a partir del cambio en el uso del suelo, conforme se transforman las necesidades de la población que habita sobre los espacios públicos del sector (ver tabla 2).

Tabla 2. Elementos de caracterización socio-espacial sobre la zona El Tintal.

\begin{tabular}{|c|c|}
\hline Aspecto & Elementos descriptores \\
\hline 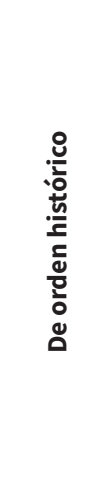 & $\begin{array}{l}\text { - Hace } 2700 \text { años aproximadamente: zona para la caza y la obtención de tintes para tejidos (Árbol de Tintal). } \\
\text { - Siglos XVI al XVIII: parcelación de tierras para la ganadería. } \\
\text { - Siglo xVIII y XIX: construcción del Seminario y Colegio Real de San Bartolomé (Orden Jesuitas 1729-1736). Creación de las } \\
\text { haciendas Tagaste y El Tintal (1869-1890). } \\
\text { - Siglo xx (Primera etapa de urbanización): construcción Aeropuerto de Techo (1929-1938) y Avenida Paseo de las Américas } \\
\text { (1935). Aparecen barrios como Patio Bonito, Abastos, María Paz y Valladolid (entre } 1974 \text { y 1990). Instalación de la planta } \\
\text { compactadora de basuras Gibraltar (1975-1993) y construcción de la Avenida Ciudad de Cali (1989). } \\
\text { - Siglo xxı (Segunda etapa de urbanización): construcción del Parque Metropolitano y la Biblioteca Pública El Tintal (2001-2003). } \\
\text { Aparición de Urbanizaciones Tintalá, Santa Fe del Tintal, Nueva Castilla (2002-2007). Construcción Centro Comercial Tintal } \\
\text { Plaza (2004) y Universidad Agustiniana (2009). }\end{array}$ \\
\hline 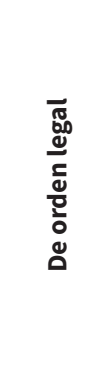 & $\begin{array}{l}\text { Recuperación ambiental y del espacio público } \\
\text { - Decreto } 738 \text { de } 2001 \text { (construcción del Parque Urbano Biblioteca El Tintal). } \\
\text { Plan de Ordenamiento Territorial de Bogotá } \\
\text { - Arts. } 25 \text { y 26, Decreto } 469 \text { de } 2003 \text { (implementación de operaciones estratégicas para mejorar las condiciones ambientales y } \\
\text { de habitabilidad). } \\
\text { - Art. } 71 \text {, Decreto } 190 \text { de } 2004 \text { (creación de la Centralidad Corabastos para estimular el desarrollo económico y la oferta de } \\
\text { servicios culturales, recreativos y ambientales en el sector). }\end{array}$ \\
\hline 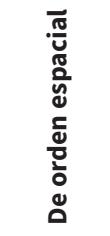 & $\begin{array}{l}\text { - Espacios abiertos que facilitan el libre acceso de la población y la realización de múltiples actividades. } \\
\text { - Hito de referencia para los habitantes del sector. } \\
\text { - No posee elementos físicos que condicionen las acciones de la población. }\end{array}$ \\
\hline
\end{tabular}

Fuente: elaboración propia. 
A partir de los cambios descritos puede establecerse que la condición pública de estos espacios es resultado de una construcción social en la que las acciones humanas, mediadas por la presencia de un conocimiento compartido, generan patrones culturales de sociabilidad (Giddens, 1993, citado en Baert, 2001), que van configurando el espacio a través del desarrollo de prácticas sociales. Estas se presentan como una forma particular de interacción con el mundo cargadas de un significado específico para los individuos que las realizan.

Desde tal perspectiva, en la zona de estudio acontecen múltiples prácticas condicionadas por la presencia de una variedad de sujetos-habitantes $^{6}$, encargados de configurar estos espacios en función del tipo de experiencias particulares; con las cuales se identifican y disponen la forma de habitar la ciudad, teniendo presente la temporalidad en la que se efectúa el tipo de servicios que se ofertan a la población (ver tabla 3).

Tales características identificadas en la zona permitieron develar varios elementos comunes que merecen ser analizados con detenimiento. En primer lugar, se establece que el desarrollo de prácticas sociales se encuentra asociado a las formas de ocupación del espacio y los flujos que allí se generan (ver mapa 2), y se constituye una conexión entre los seres humanos que las realizan y las zonas concretas donde se materializan.

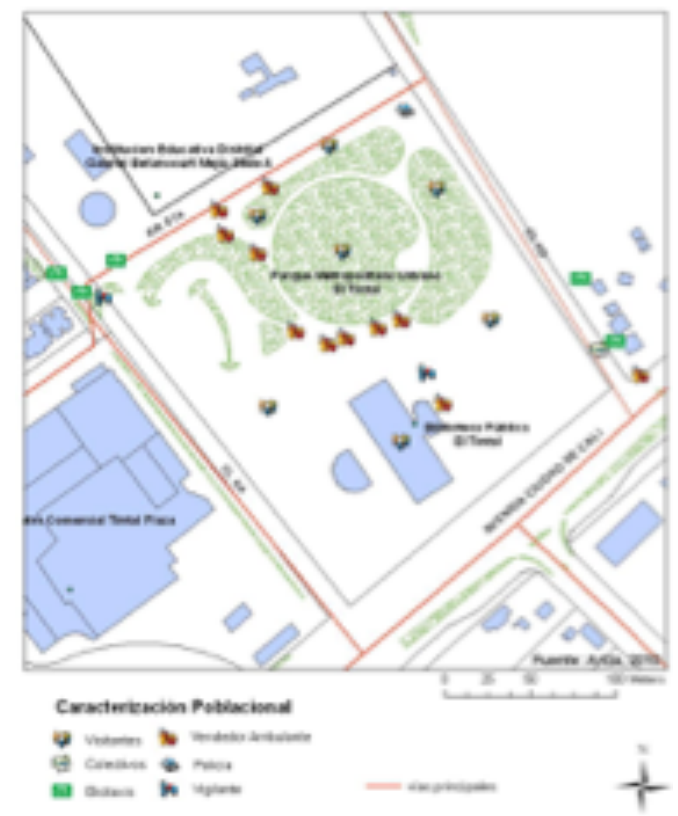

Mapa 2. Distribución poblacional de los sujetos en torno a los espacios públicos del parque y la Biblioteca El Tintal.

Fuente: elaboración propia.

6 La categoría de sujeto-babitante es desarrollada por Alicia Lindón (2009a) al considerar que el sujeto es el principal constructor de lo social y lo urbano en las ciudades; el cual, a partir del desarrollo de su espacialidad desde la base de la experiencia, las emociones y la corporalidad, concibe y percibe el mundo hasta interiorizarlo como lugar.
En segundo lugar, el desarrollo de prácticas sociales sobre los espacios públicos lleva implícita la interacción como mecanismo para la construcción de significados particulares en función del ser humano que los utiliza, y que contribuyen a forjar una experiencia espacial determinada para cada sujeto. En la medida que los procesos de interacción permiten establecer conexiones de cercanía hacia los otros, los seres humanos paulatinamente van encontrando su lugar en el mundo; como resultado no solo de la apropiación individual, sino también de la apropiación colectiva de ese espacio, mediante las formas como se concibe, interpreta y utiliza el espacio público y el carácter social que implica construirlo ${ }^{7}$.
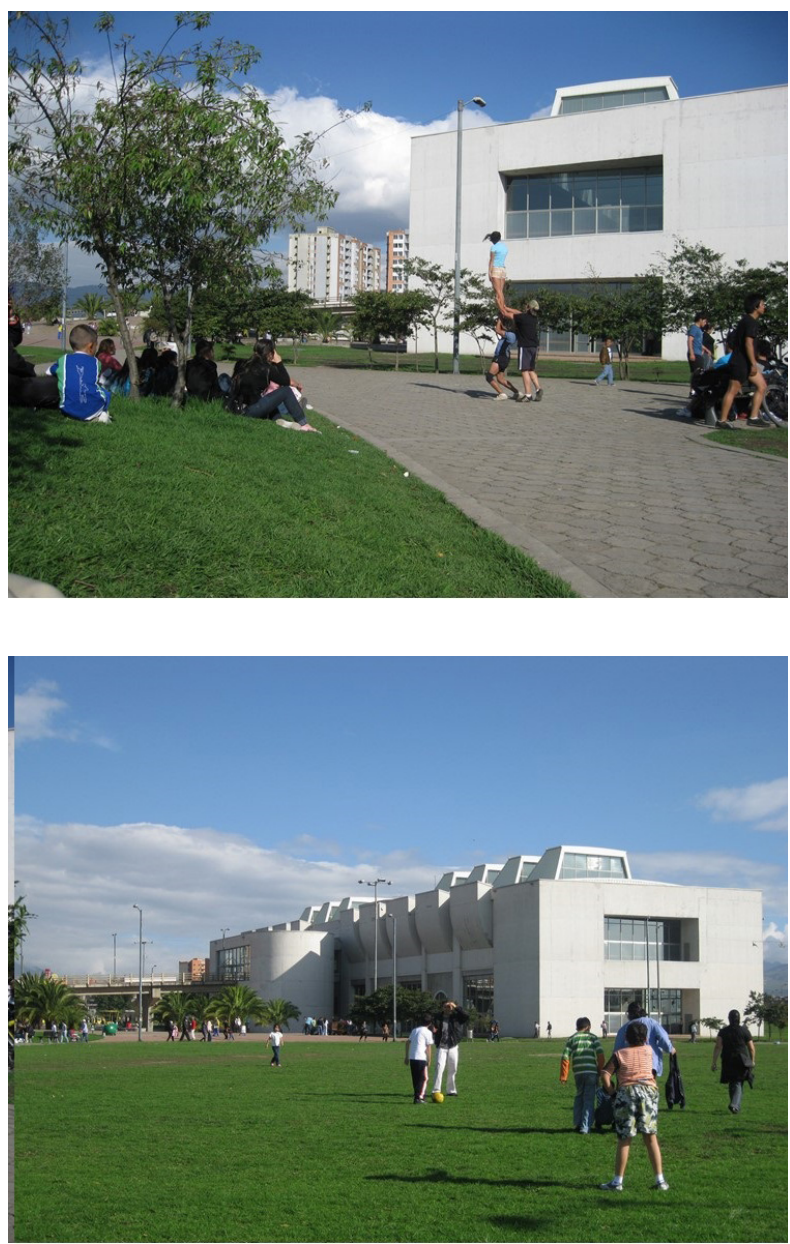

Fotografías 1 y 2. Las zonas verdes: espacios de mayor confluencia poblacional en los espacios públicos de El Tintal.

Fuente: tomadas por el autor.

7 De esta forma, el mismo ser humano se reconoce como ser en el mundo, como parte integrada del espacio que ocupa y donde construye y define su propio topos, su lugar natural en el mundo (Yory, 2007). 
Tabla 3. Categorización de actividades en los espacios públicos de El Tintal.

\section{Actividades realizadas en El Tintal según la temporalidad y la oferta de servicios disponibles para la población}

Siglas de referencia: (OE): Oferta educativa, (OC): Oferta cultural, (OD): Oferta deportiva, (OR): Oferta recreativa, (OS): Oferta de servicios.

\begin{tabular}{|c|c|c|c|c|}
\hline Espacio & \multicolumn{2}{|c|}{ Biblioteca } & \multicolumn{2}{|c|}{ Parque } \\
\hline Temporalidad & Entre semana & Fin de semana & Entre semana & Fin de semana \\
\hline \multirow[b]{2}{*}{ Mañana } & \multicolumn{2}{|c|}{ Consulta de material bibliográfico (OE). } & \multirow{2}{*}{$\begin{array}{l}\text { Actividad física: trotar, montar } \\
\text { bicicleta (OD). } \\
\text { Tránsito peatonal hacia los flujos de } \\
\text { transporte (OS). }\end{array}$} & \multirow{2}{*}{$\begin{array}{l}\text { Actividad física: trotar, montar } \\
\text { bicicleta, jugar fútbol (OD). } \\
\text { Flujos de transporte (OS). } \\
\text { Aérobicos y juegos para menores de } \\
\text { edad (OR). }\end{array}$} \\
\hline & $\begin{array}{l}\text { Visitas guiadas para } \\
\text { colegios (OE). }\end{array}$ & $\begin{array}{l}\text { Talleres de lectura y } \\
\text { presentaciones de cine (oc). }\end{array}$ & & \\
\hline \multirow[b]{2}{*}{ Tarde } & \multicolumn{2}{|c|}{ Consulta de material bibliográfico (OE). } & \multirow[b]{2}{*}{$\begin{array}{l}\text { Ventas informales (OS). } \\
\text { Recreación pasiva: caminar o } \\
\text { descansar por el parque (OR). } \\
\text { Actividad física: montar bicicleta (OD). }\end{array}$} & \multirow[b]{2}{*}{$\begin{array}{l}\text { Ventas informales (OS). } \\
\text { Recreación pasiva: caminar o } \\
\text { descansar por el parque (OR). } \\
\text { Actividad física: bicicleta y fútbol (OD). }\end{array}$} \\
\hline & $\begin{array}{l}\text { Talleres de creación } \\
\text { artística y literaria (oc). }\end{array}$ & $\begin{array}{l}\text { Exposiciones artísticas y } \\
\text { oferta de conciertos (oc). }\end{array}$ & & \\
\hline Noche & $\begin{array}{l}\text { Conferencias y } \\
\text { conciertos (oc). }\end{array}$ & No aplica. & \multicolumn{2}{|c|}{$\begin{array}{l}\text { Tránsito peatonal y flujos de transporte (os). } \\
\text { Consumo de licor, drogas y otras actividades ilícitas. }\end{array}$} \\
\hline $\begin{array}{l}\text { Fechas y eventos } \\
\text { especiales }\end{array}$ & \multicolumn{2}{|c|}{$\begin{array}{l}\text { Meses diciembre-enero y junio-julio: actividades de } \\
\text { Bibliovacaciones enfocadas a niños, niñas y jóvenes } \\
\text { (OE/OC). }\end{array}$} & \multicolumn{2}{|c|}{$\begin{array}{l}\text { Agosto: Festival de Cometas, Feria Empresarial Local y Fiestas Patrias (OR/OS/OC). } \\
\text { Noviembre y diciembre: iluminación navideña y novena de aguinaldos (Ruta de } \\
\text { la Navidad) / Astronomía al Parque (OR/OC). }\end{array}$} \\
\hline
\end{tabular}

Fuente: elaboración propia. 


\section{El Tintal: un espacio que se construye socialmente a partir de la experiencia espacial}

A partir de la reflexión en torno a las manifestaciones y las transformaciones de la vida pública, en escenarios donde confluyen la diversidad, la diferencia y la pluralidad de subjetividades intervinientes en la construcción de una trama de la vida cotidiana ${ }^{8}$, se pudieron establecer relaciones entre las prácticas sociales y la apropiación del espacio público sobre El Tintal desde 3 ámbitos, que reconocen factores incidentes en la construcción social de los espacios públicos a través de diferentes actos del habitar:

\section{a) El espacio público como lugar:}

Cada sujeto-habitante en El Tintal configura sus prácticas según los ritmos, flujos y acciones que se producen en los espacios que habita. Esto le permite identificar condiciones favorables para el desarrollo de actividades a partir de sus experiencias cotidianas. Esta significación hacia lugares cercanos a la subjetividad-isotopías-, junto con el reconocimiento de lugares lejanos -heterotopías- (Lefebvre, 1972), permite que El Tintal sea identificado, desde el reconocimiento y comprensión, hacia las posibilidades de acción y expresión en los sujetos; las cuales son producidas en escenarios que generan condiciones apropiadas para la constitución de una vida cotidiana.

La interacción entre sujetos-habitantes contribuye a desarrollar formas de apropiación colectiva hacia el espacio público, identificando y reconociendo objetos y sujetos que intervienen en la realización de actividades cotidianas (caminar, trabajar, hacer deporte y trasladarse al trabajo o a la vivienda). Consolidar estas formas de acción como prácticas sociales permite forjar significados particulares de ser en el mundo para cada sujeto, donde identificarse, pertenecer, apropiarse y corresponderse como parte integrada del espacio que se ocupa, implica una entrada en propiedad hacia el espacio público.

Los significados hacia el escenario de lo público producidos por los sujetos-habitantes que actúan y ejercen un acto de presencia rescatan en el sector funciones de tipo recreativo, de esparcimiento y de movilidad para la ciudad. Además de ello, la zona presentada les ofrece varios servicios, articulados con el desarrollo de sus actividades (placenteras o laborales), constituyéndose como punto de referencia socioespacial tanto a nivel comercial como de descanso y esparcimiento.

8 El concepto de trama de la vida cotidiana es desarrollado por Alicia Lindón (1999) para referirse a los vínculos entre los roles, las funciones y las relaciones sociales que desarrolla el sujeto en conexión permanente con los fondos espaciales y temporales, sobre los cuales determinadas actividades se materializan.

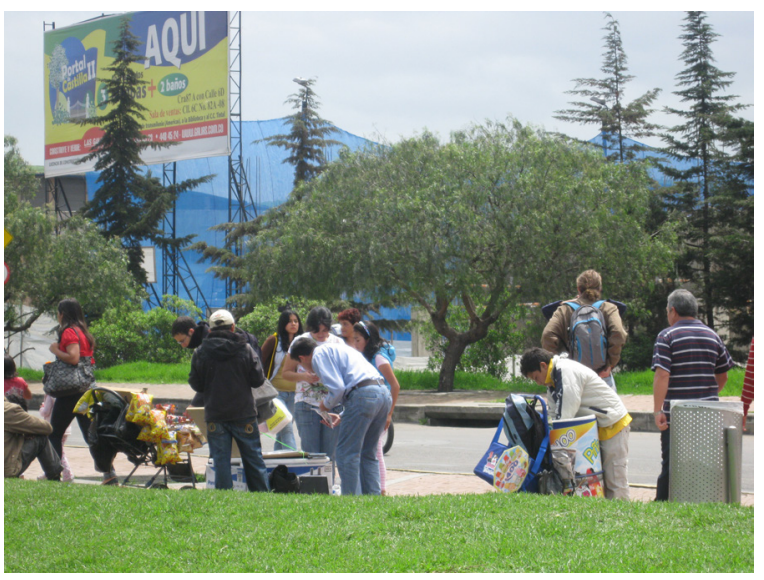

Fotografía 3. Confluencia de actividades realizadas por los sujetos-habitantes de El Tintal.

Fuente: tomadas por el autor.

Dichas apreciaciones de los sujetos-habitantes establecen la importancia de los espacios públicos de El Tintal como escenarios que conectan a los lugares a través de relaciones intersubjetivas, mediante prácticas sociales que reúnen a sujetos con experiencias y cotidianidades distintas. La conjunción de subjetividades sobre el espacio público habitado es lo que permite comprender que toda práctica social se relaciona estrechamente con lo que implica vivir en la ciudad. El Tintal, en este caso particular, se constituye en un espacio del habitar al permitirle a los sujetos protagonistas identificarse con objetos y con otros actores allí presentes que intervienen en sus actividades cotidianas desde la realización de prácticas sociales.

\section{b. Prácticas sociales como expresión de ciudadanía:}

Toda práctica espacial desplegada por cada sujeto se encuentra en constante relación con las prácticas del otro, y se establecen así convergencias a partir de las cuales se estructura y edifica la práctica social. El intercambio de información, sentidos y experiencias entre los sujetos que interactúan sobre un espacio en particular conduce a una construcción socioespacial y son las prácticas las que permiten develar la forma como se establecen relaciones de cercanía o proximidad.

Al momento de indagar sobre las apreciaciones subjetivas por las cuales se reconocen, identifican y significan los espacios públicos de El Tintal, se destaca un reconocimiento por parte de los sujetos-habitantes de otros actores con los cuales se interactúa al momento de realizar determinada práctica social. De aquí se establece que los sujetos no solo construyen una manera particular de representar los espacios que habitan a partir de sus intereses, sino que involucran como parte de sus actividades cotidianas a otros individuos, que de manera directa o indirecta intervienen en el desarrollo de su vida cotidiana (ver mapa 3). 


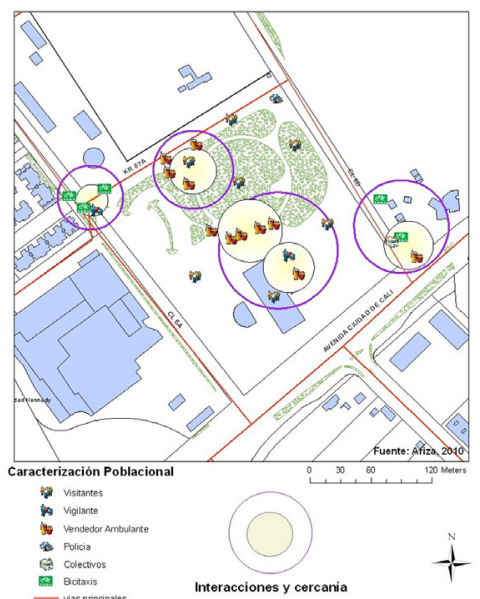

Mapa 3. Interacción y cercanía entre sujetos-habitantes de El Tintal ${ }^{9}$. Fuente: elaboración propia.

Para los sujetos-habitantes, los espacios públicos están hechos para el descanso y la relajación de los visitantes, por ello sus acciones están encaminadas a garantizar su cuidado, preservación y seguridad. Tales apreciaciones emergen solo cuando el sujeto se asume como parte del lugar que habita al generar las condiciones que garanticen su estar en el mundo, mediante procesos de apropiación y pertenencia hacia el espacio. Desarrollar reflexividad hacia la práctica cotidiana permite que el sujeto cree conciencia sobre sus propias acciones y sobre las acciones de los otros, con los cuales interactúa (Giddens, 1993, citado en Baert, 2001; Sánchez, 2007). Las prácticas sociales que acontecen sobre el espacio público tienen una connotación ciudadana, ya que reflejan procesos de apropiación consciente que permiten identificar ciertas acciones que transgreden lo permitido o lo deseable para el beneficio y el aprovechamiento de los elementos que se ofrecen en la zona.

La idea de ciudadanía presente entre los sujetos-habitantes puede entenderse desde dos referentes:

- Las interacciones sociales se encuentran en conexión con las percepciones y los modos de apropiación que los sujetos realizan sobre el espacio. El resultado de esta conjugación se manifiesta en la regulación de actividades entre individuos y en espacios que son socialmente compartidos, y sobre los cuales se crean pautas que condicionan las conductas de los sujetos (Lindón, 2009a).

- Las prácticas sociales son el resultado de la articulación entre posibilidades y prohibiciones para la acción, distinguiéndose así una separación entre lo que es considerado como reprochable y

9 Los círculos representan la cercanía entre personas en diferentes zonas de confluencia en el espacio público. las conductas socialmente aceptadas. Cada sujeto-habitante debe hacer uso de un acervo de conocimientos construidos a partir de la reflexión sobre su propia acción social; razón por la cual ocurre una interiorización de reglas sociales que estipulan y encaminan la vida social en cuanto a procedimientos específicos de cómo desarrollar la vida cotidiana en contextos de sociabilidad (Giddens, 1993, citado en Baert, 2001).

En la medida en que los sujetos edifican sus prácticas sociales a partir de la forma como ocupan y habitan los espacios públicos, sus acciones se configuran de forma consciente teniendo en cuenta las implicaciones o consecuencias que puedan generar hacia los otros (ver mapa 4). En este sentido,

[...] la confluencia de significados y funciones diversos en escenarios de sociabilidad permite visibilizar los problemas de la vida en la ciudad y las posibilidades de transformarlos en oportunidades que promuevan acciones encaminadas a mejorar la calidad de vida en sus habitantes [...]. (Ramírez, 2003, p.37).

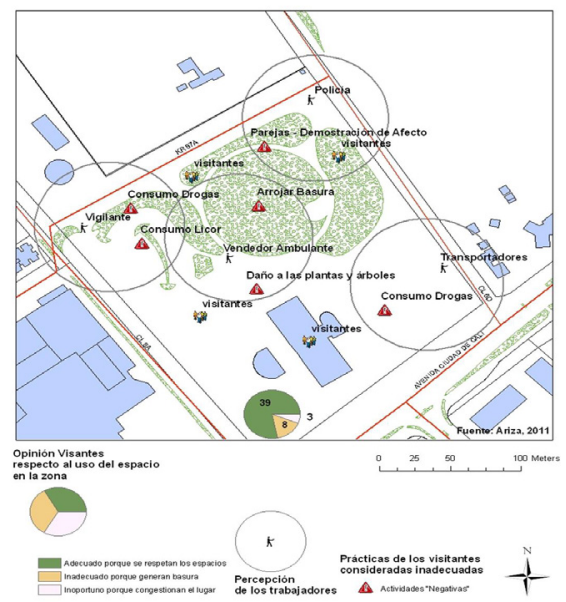

Mapa 4. Percepción ciudadana en torno a actividades que se desarrollan en los espacios públicos de El Tintal.

Fuente: elaboración propia.

c) Prácticas sociales en el espacio público como transformadoras de la realidad social:

La capacidad de transformar la realidad social está mediada por la incidencia que tengan determinadas acciones sobre la conducta de los sujetos, lo cual permite un reconocimiento de sí mismo y de los otros. Cuando determinada práctica se hace recurrente como el caso de la prestación del servicio de transporte informal o la realización de actividades deportivas, se producen transformaciones tanto en el espacio físico como en el conocimiento social mediante la generación de conductas específicas para cada actividad en particular. 
El cambio en la realidad social viene dado por la forma como se involucran conocimientos respecto al modo de actuar entre individuos, es así como entran en diálogo diversas estrategias y tácticas ${ }^{10}$ generadas desde el conocimiento que se tiene sobre el lugar o producto de su apropiación. Al efectuarse determinada práctica social entran en juego una serie de condicionamientos que amplían o limitan el campo de acción de los sujetos en contextos espaciotemporales, lo cual estipula las maneras como se interactúa con el otro a partir de dinámicas que evidencian la presencia o ausencia de poder.
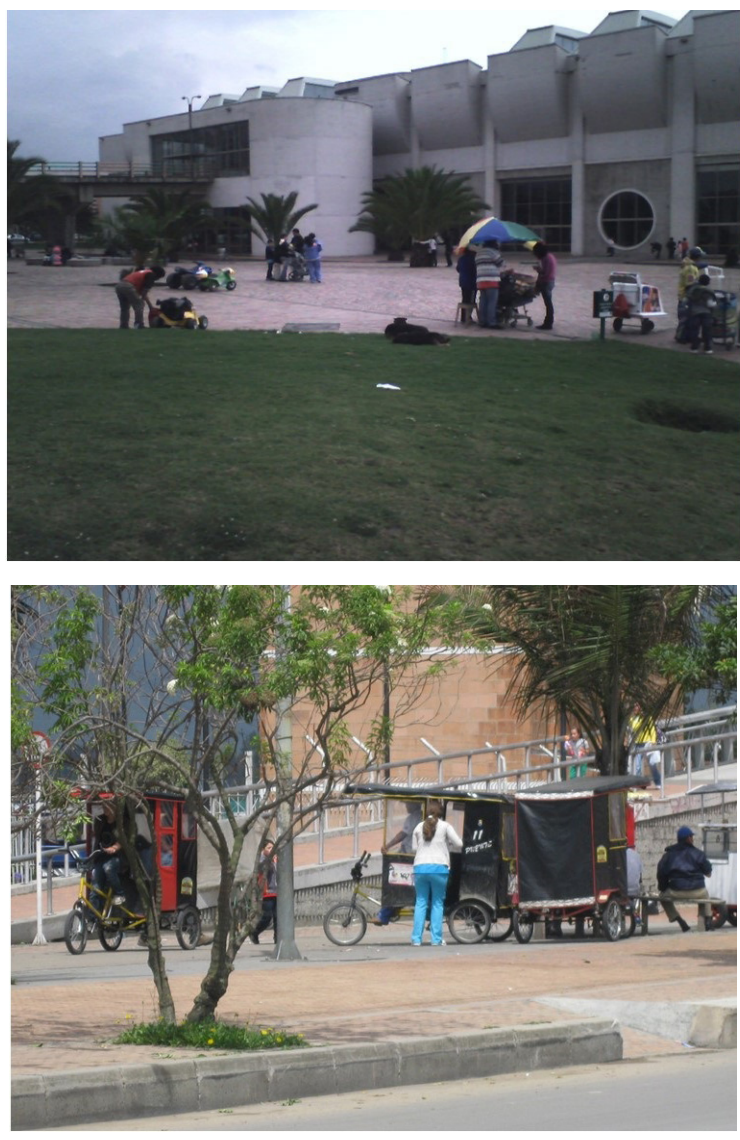

Fotografías $\mathbf{4}$ y $\mathbf{5}$. Contraste entre zonas donde se desarrollan actividades informales.

Fuente: tomadas por el autor.
La forma como se establecen limitantes a la acción viene dada por el conocimiento previo sobre las zonas en las que se pueden o no desarrollar ciertas prácticas. La experiencia espacial es un elemento primordial para reafirmar que la construcción de lugares se vincula con el desarrollo de territorialidades que expresan la pertenencia y la apropiación hacia el espacio, lo cual es el resultado de la capacidad del sujeto para cambiar situaciones que afectan el desarrollo de sus actividades cotidianas en situaciones favorables para sí. Esto conduce a afirmar que cada sujeto se encarga de articular sus prácticas cotidianas conforme le es posible actuar en espacios, contextos y situaciones específicas, teniendo presentes unos mínimos necesarios para la convivencia social a partir del respeto de unos compromisos generados en el ambiente social sobre el cual se desenvuelve (ver mapa 5).

Estos principios de negociación son los que permiten que se logre pasar de una práctica personal a una práctica intersubjetiva sobre el espacio (Sánchez, 2007), ya que se establecen puntos en común para configurar todo el sistema de conocimientos, valores y actitudes, sobre los cuales tiene lugar la realización de intercambios sociales. Por tal motivo, apropiarse del espacio público se encuentra en estrecha relación con la transformación de un orden urbano a partir de la aparición de nuevas experiencias y usos sociales (Ramírez y Aguilar, 2006), establecidos por la emergencia de estrategias y las tácticas para la acción del sujeto en situaciones específicas, que están encaminadas a garantizar un beneficio particular junto con la convivencia social, a partir del respeto por las prácticas de otros (De Certeau, 1996).

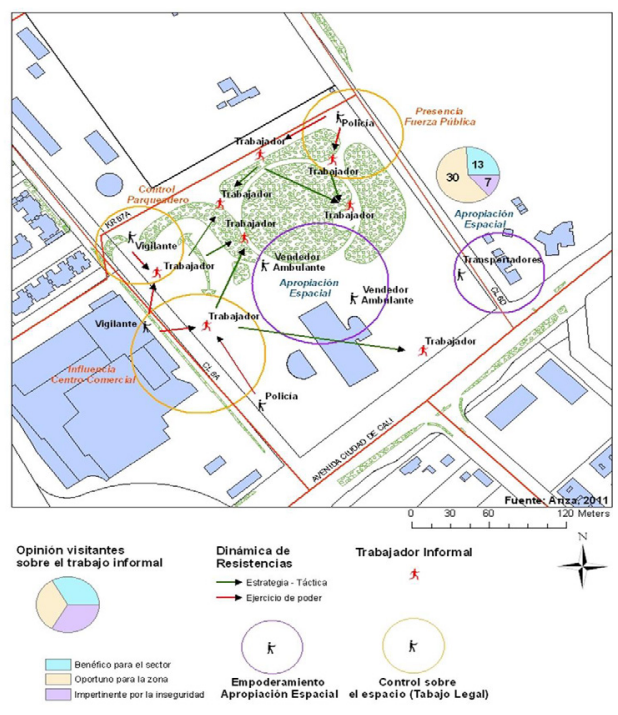

Mapa 5. Territorialización de los espacios públicos en El Tintal y representación de formas de resistencia frente a la ocupación de los espacios. Fuente: elaboración propia. 10 Los conceptos de estrategia y táctica son desarrollados por Michel De Certeau
(1996), y representan las formas como se estructuran las prácticas sociales a partir de la mediación entre las capacidades para la acción del individuo y las posibilidades para la acción en campo del otro. Es así como se establecen parámetros a través de los cuales se regulan las acciones de los individuos en el desarrollo de prácticas cotidianas. 


\section{Conclusiones}

Tras realizar un balance frente al proceso investigativo se puede concluir que El Tintal es un espacio público que se construye socialmente a partir de la experiencia espacial, debido a la importancia que otorgan los sujetos-habitantes al sector y a los espacios públicos a través de sus percepciones. Por sus características, la zona se convierte en una nueva centralidad para la ciudad de Bogotá al incidir tanto en el crecimiento poblacional, mediante la planificación urbana, como en el desarrollo económico del sector, al permitir una diversificación laboral.

El Tintal evidencia un caso representativo de este crecimiento urbano en la medida en que el proceso de urbanización ha permitido el emplazamiento no solo de complejos residenciales unifamiliares, sino de grandes proyectos de propiedad horizontal establecidos para solventar la necesidad de habitabilidad en un escenario con una marcada tendencia hacia "adecuar los huecos de la ciudad a las necesidades de la población” (Tardin, 2006, p. 396).

La emergencia de espacios públicos como este va de la mano con la transformación en la calidad de vida de sus habitantes. Esto promueve aspectos como la recuperación ambiental, el ordenamiento planificado de la urbanización y la oferta de escenarios para el encuentro social de la población. Igualmente, la transformación espacial incide en la aparición de nuevos actores y prácticas (trabajo informal, principalmente), como respuesta a una cobertura insuficiente de servicios dentro de la oferta recreativa, educativa y cultural de la ciudad. Esto conduce a interpretar que las acciones cotidianas de los sujetos-habitantes suceden como formas de empoderamiento ${ }^{11}$ (Sánchez, 2002) en las cuales intervienen procesos de apropiación individual o colectiva del espacio, generando así nuevas experiencias socioespaciales.

Cada forma de experienciar El Tintal como un espacio público vivido (Ramírez y Aguilar, 2006) lleva implícita la idea de una construcción social de la ciudad, donde a partir de la subjetividad se construyen formas de habitar desde el quehacer de la vida cotidiana. En este sentido, el aporte que realiza el presente trabajo para el conocimiento social radica en descubrir, representar y visibilizar terraes cognitaes e incognitaes ${ }^{12}$ (Lindón, 2009b) provenientes de la experiencia espacial particular de cada sujeto. Estas involucran tras de sí un mundo complejo de relaciones en las que intervienen significados colectivos acerca de cómo se concibe, interpreta, apropia y transforma el espacio construido socialmente.

\footnotetext{
11 Cada proceso de apropiación espacial se materializa a través del empoderamiento que permite a los sujetos hacer conciencia sobre sus necesidades y los recursos para suplirlas, las posibilidades y limitaciones presentes en los lugares habitados que favorecen o dificultan su desarrollo social en contextos de interacción permanente. Se trata de un proceso intencional, intersubjetivo y continuo de conversión de individuos en sujetos conscientes de sí mismos, de las circunstancias y del entorno social donde habitan; quienes a través de la acción comprensiva y crítica transforman las condiciones de realización para sus propias interacciones sociales (Sánchez, 2002).

12 Las categorías de terrae connitae y terrae incognitae son trabajadas por Alicia Lindón (2009b) a partir de los trabajos de Jhon K. Wright (1947) sobre el significado de las geografías personales en la estructuración de una cognición espacial. En tal sentido, una terrae cognitae es aquella que se presenta como cercana y familiar al individuo y sobre la cual este posee cierta información y conocimiento, que le permite actuar libremente y con total control de las acciones que realiza. Lo opuesto a ello es la terrae incognitae, pues es aquel control de las acciones que realiza. Lo opuesto a ello es la terrae incognitae, pues es aquel
espacio que se encuentra lejano a la cotidianidad del sujeto y sobre el cual se generan sentimientos de incertidumbre, desconfianza o temor hacia lo desconocido. En ambos casos, la articulación entre terrae cognitae y terrae incognitae determina la conformación de una experiencia espacial subjetiva propia para cada individuo.
}

\section{Referencias}

Arendt, H. (1993). La condición humana. Barcelona, España: Paidós.

Baert, P. (2001). La teoría social en el siglo XX. Madrid, España: Alianza.

Bonilla, E. y Rodríguez, P. (1995). Más allá del dilema de los métodos: la investigación en ciencias sociales. Bogotá, Colombia: Universidad de los Andes, CEDE.

Castells, M. (1976). La cuestión urbana. Ciudad de México, México: Siglo XXI.

De Certeau, M. (1996). La invención de lo cotidiano I. Artes de hacer. Ciudad de México, México: Universidad Iberoamericana.

Decreto 738 de 2001. "Por el cual se adopta el Plan Maestro del parque urbano Biblioteca El Tintal”. 21 de septiembre de 2001. Alcaldía Mayor de Bogotá. Bogotá, Colombia

Decreto 190 de 2004, Artículo. 71. "Por medio del cual se compilan las disposiciones contenidas en los Decretos Distritales 619 de 2000 y 469 de 2003.". Alcaldía Mayor de Bogotá, Bogotá Colombia.

Decreto 469 de 2003. Artículos 25 y 26. "Por el cual se revisa el Plan de Ordenamiento Territorial de Bogotá D.C.". Alcaldía Mayor de Bogotá, Bogotá, Colombia.

Giddens, A. (1990). Teoría de la estructuración y praxis social. En A. Giddens (Comp.), La teoría social, hoy, (pp. 351-393). Madrid, España: Alianza.

Lefebvre, H. (1972). La revolución urbana. Barcelona, España: Alianza.

Lindón, A. (1999). De la trama de la cotidianidad a los modos de vida urbanos. El Valle de Chalco. Ciudad de México, México: El Colegio de México.

Lindón, A. (2009a). La construcción socioespacial de la ciudad: el sujeto cuerpo y el sujeto sentimiento. Revista Latinoamericana de Estudios sobre Cuerpos, Emociones y Sociedad, (1), 6-20.

Lindón, A. (2009b). La educación geográfica y la ampliación de las Terrae Cognitae personales. En M. Garrido (Ed.), La espesura del lugar: reflexiones sobre el espacio en el mundo educativo, (pp. 153-170). Santiago de Chile, Chile: Universidad Academia de Humanismo Cristiano.

Lindón, A. (2010). Invirtiendo el punto de vista: las geografías urbanas holográficas del sujeto habitante. En A. Lindón y D. Hiernaux (Dirs.), Los giros de la geografía humana. Desafíos y horizontes (pp. 175-200). Barcelona, España: Anthropos.

López, H. (1998). La metodología de la encuesta. En L. Galindo (Coord.), Técnicas de investigación en sociedad, cultura y comunicación, (pp. 33-73). México: Pearson. 
Páramo, P. (2007). El significado de los lugares públicos para la gente de Bogotá. Bogotá, Colombia: Universidad Pedagógica Nacional.

Ramírez, P. (2003). Espacio público: ciudad y ciudadanía. De los conceptos a los problemas de la vida pública local. En P. Ramírez (Coord.), Espacio público y reconstrucción de ciudadanía, (pp. 31-58). Ciudad de México, México: Facultad Latinoamericana de Ciencias Sociales.

Ramírez, P. y Aguilar, M. (Coords.). (2006). Pensar y habitar la ciudad. Afectividad, memoria y significado en el espacio urbano contemporáneo. Barcelona, España: Anthropos.

Sánchez, A. (2002). Dispositivos de empoderamiento para el desarrollo psicosocial. Universitas Psychologica, 1(2), 39-48.
Sánchez, A. (2007). Gestión social, sujeto y participación. El espacio público como escenario de constitución del sujeto político. En C. Yory (Ed.), Espacio Público y formación de ciudadanía, (pp. 45-74). Bogotá, Colombia: Pontificia Universidad Javeriana.

Tardin, R. (2006). La ciudad informal. En J. Nogué y J. Romero (Eds.), Las otras geografías, (pp. 389-404). Valencia, España: Tirant lo Blanch.

Valencia, M. (2009). Cartografías urbanas. Imaginarios, huellas y mapas. Revista Electrónica DU\&P. 6(16). Recuperado de: http://www.ucentral.cl/du\&p/pdf/16_huellas_imaginarios.pdf

Yory, C. (2007). Topofilia o la dimensión poética del habitar. Bogotá, Colombia: Pontificia Universidad Javeriana. 